\title{
Programa "ciência vai à escola" - museu de ciências naturais da ufpr: construindo uma visão de ciência na educação básica
}

\author{
Victor E. Pauliv \\ Luciana C. Carvalho \\ Camilla Felippe \\ Rafaela Bobato \\ Fernando A. Sedor
}

\begin{abstract}
Resumo
O Programa Ciência Vai à Escola (PCVAE) foi criado em 1997 em uma parceria entre o Museu de Ciências Naturais (MCN-SCB-UFPR) e Departamento de Genética da Universidade Federal do Paraná em conjunto com a Sociedade Brasileira para o Progresso da Ciência, Regional Paraná, tendo como pilares a educação para a ciência e a popularização, alfabetização e divulgação científica. $O$ PCVAE busca aproximar a universidade das escolas de educação básica, contribuir para modificar as estruturas da educação científica brasileira e inserir o conhecimento científico no cotidiano de estudantes, de professores e da comunidade em geral. O Programa é desenvolvido através de exposições itinerantes, palestras, cursos e oficinas em universidades, colégios, praças e outros ambientes seguindo as seguintes etapas: observação, reflexão, formulação e teste de hipóteses e experimentação. As atividades também abrangem a exposição permanente "Os Seres Vivos no Ensino Fundamental e Médio" do MCN-SCB-UFPR com visitações monitoradas; confecção de modelos didáticos e coleções de material biológico para empréstimo; orientação acadêmica e de professores, entre outras. O planejamento destas ações envolve pesquisa, estudo de assuntos científicos e educacionais, preparação do material, execução e realização de avaliações contínuas e periódicas para verificar a efetividade do PCVAE. Durante os 15 anos de atuação o PCVAE junto ao MCN-SCB-UFPR atingiu mais de 120 mil participantes de cerca de 420 instituições diferentes, contribuindo significativamente nos seus objetivos de educar, alfabetizar, popularizar e divulgar a ciência.
\end{abstract}

Palavras-chave: Educação para a Ciência, Popularização científica e Divulgação da Ciência.

\section{Abstract}

The Program "Ciências Vai à Escola" (PCVAE) was created in 1997 in a partnership between the "Museu de Ciências Naturais" (MCN-SCB-UFPR) and Department of Genetics of the Federal University of Paraná and the Brazilian Association for the Advancement of Science ("Sociedade Brasileira para o Progresso da Ciência") having the Sciences education and scientific popularization, literacy and divulgence as pillars. The PCVAE tries to approximate the university from basic education schools, contribute to modify the structures of Sciences education in Brazil and insertion of scientific knowledge in the life of students, teachers and the community in general. The program is developed through 
itinerant exhibitions, lectures workshops at universities, colleges, squares and other places by following these steps: observation, reflection, formulation and testing of hypothesis and experimentation. The activities also includes the permanent exhibition "Os Seres Vivos no Ensino Fundamental e Médio" ("Living Creatures in Elementary and Secondary Education") of the MCN-SCB-UFPR with monitored visitation, the elaboration of didactic models and biological collections for loan; academic advising and teachers guidance, among others. The planning of these actions involves scientific and educational research, material preparation, execution and implementation of continuous and periodic evaluations to verify the effectiveness of PCVAE. During the 15 years of operation the PCVAE reached approximately 120 thousands of participants of almost 420 different institutions and questionnaires show that less than $10 \%$ had visited a museum, reflecting a significant contribution to the knowledge acquisition of the participants and reaching the goals of Science education, literacy, popularization and divulgation every day.

Key words: Scientific education, Scientific popularization and Sciences divulgation.

\section{Introdução}

O progresso da Ciência e da Tecnologia é evidente nos dias atuais, de modo que se torna impossível ficar alheio aos acontecimentos relacionados a estes temas. Todavia, o aumento do conhecimento gerado pela pesquisa científica, não garante o repasse dessas informações para que a população tenha acesso aos resultados de forma efetiva. O contato superficial proporcionado pelos meios de comunicação como rádio, televisão, jornais e revistas que são mais acessíveis à população em geral, não possibilita um claro entendimento do que consiste o conhecimento científico e como ele é gerado. A dificuldade de realizar o repasse da informação não está só na divulgação realizada pelos meios de comunicação, mas sim na ausência de uma alfabetização científica fornecida na educação básica.

No mundo contemporâneo, apesar do público em geral ter facilidade de acesso aos resultados de pesquisas que abordam temas que influenciam diretamente suas vidas (e.g. doenças causadas por vírus e bactérias, células tronco, mudanças climáticas entre outros temas recorrentes) a divulgação nem sempre atinge o objetivo de esclarecer dúvidas da população. Em muitas situações causa efeito contrário, acarretando interpretações errôneas, que impactam negativamente suas rotinas ocasionando um afastamento do conhecimento científico.

Apesar da divulgação por parte da mídia ser importantíssima, o objetivo de esclarecimento só é alcançado quando o público está apto a compreender as informações que tem acesso e utilizar as que são relevantes para o seu dia a dia.

Baseando-se nessa problemática, foi criado em 26 de abril de 1994 o Museu de Ciências Naturais do Setor de Ciências Biológicas da Universidade Federal do Paraná (MCN-SBC-UFPR) com o intuito de realizar a divulgação científica sem banalizá-la e atuar na formação inicial e 
continuada de professores da rede Estadual de Ensino. Posteriormente, a partir de uma perspectiva científico-educativa, o Programa "Ciência Vai à Escola" (PCVAE) foi criado em 1997, por iniciativa do Prof. Euclides Fontoura da Silva Júnior do MCN-SCB-UFPR em parceria com o Departamento de Genética da UFPR e a Sociedade Brasileira para o Progresso da Ciência (SBPC Regional do Paraná) e continua atuante até os dias atuais.

O PCVAE apresenta como pilares: a educação para a Ciência, popularização, alfabetização e divulgação científica. O propósito do PCVAE é contribuir com a qualidade do ensino de Ciências e Biologia nas instituições de ensino, com a finalidade de modificar as estruturas da educação científica brasileira, pautando-a na formação da cidadania dentro dos princípios da educação e alfabetização para a Ciência. O PCVAE propõe-se a construir uma visão de Ciência entre estudantes e professores condizente com o progresso científico e tecnológico da sociedade, melhorando o ensino de Ciências e Biologia, uma vez que segundo Silva-Junior (2007) "quando se educa para Ciência, num sentido mais amplo, não só se ensina Ciências, mas está se introduzindo uma visão de ÉTICA, de VERDADE, de RESPEITO e de SOCIEDADE".

O objetivo fundamental do PCVAE é despertar o interesse de alunos dos ensinos Fundamental e Médio, como também a Educação de Jovens a Adultos e oferecer experiências fora da rotina escolar, porém complementares às disciplinas relacionadas às Ciências Naturais, oferecendo ao público alvo um contato mais significativo com a Ciência nas escolas.

O PCVAE através de seu caráter extensionista, iniciou suas ações através de cursos de capacitação, aperfeiçoamento e proficiência para professores, no sentido de colaborar com a melhoria da formação desses profissionais e o consequente efeito multiplicador. Entretanto, no desenvolvimento desses cursos, constatou-se que apesar de haver uma melhora na compreensão dos conteúdos abordados, a correta concepção, apreensão e inter-relação de conceitos, e importância da Ciência por parte dos alunos ainda se mostravam insuficientes. Para uma maior adequação da realidade encontrada, o PCVAE foi reestruturado para que suas ações tivessem como principal foco os estudantes, sem excluir os professores e trabalhando de acordo com a concepção de FREIRE-MAIA (2007) de Ciência como um processo inacabado, em constante elaboração, ampliação e revisão.

\section{Metodologias e ações}

A metodologia adotada para o desenvolvimento das atuações do PCVAE junto ao Museu de Ciências Naturais (MCN-SCB-UFPR) incluem ações de ampliação do conhecimento científico de estudantes, professores e comunidade em geral (SILVA-JUNIOR 2001). A interatividade com os estudantes durante as atividades desenvolvidas tem como base a concepção do método científico, de tal forma que os alunos são instigados a observar o material/fenômeno envolvido com a atividade, a refletir sobre ele e desenvolver questões explicativas a respeito, assim os 
estudantes formulam hipóteses a fim de explicar os fenômenos e buscar respostas às questões levantadas previamente. Estas hipóteses são posteriormente discutidas e testadas com o auxílio de um moderador.

Esses passos são trabalhados com os participantes durante todas as atividades, a fim de reconstruir, ampliar, desmistificar conceitos e inserir o conhecimento científico no cotidiano dos alunos. Assim, utilizando a observação, a experimentação e o questionamento, é despertada a curiosidade no aluno, estimulando-o a usar o método científico na solução de problemas. A popularização da Ciência através da alfabetização científica consiste em veicular informações através de ações demonstrativas, expositivas e interativas de uma forma que a população compreenda o avanço e o desenvolvimento da Ciência (CORRÊA \& SILVA-JUNIOR, 2010).

Para o desenvolvimento dessas ações o Museu de Ciências Naturais da UFPR apresenta uma exposição permanente intitulada "Os Seres Vivos no Ensino Fundamental e Médio" que tem o propósito de oferecer infraestrutura e suporte para facilitar o aprendizado, uma vez que, possibilita o contato direto com alguns dos objetos de estudo. Esta exposição contém uma grande diversidade de organismos viventes (e.g. plantas, invertebrados e vertebrados), e extintos (fósseis). Dentre os temas da Zoologia, além de espécimes preservados em via líquida e taxidermizados, também conta-se com uma variedade de animais (peixes, anfíbios, serpentes, tartarugas e artrópodes) e plantas mantidos vivos em aquários, terrários e aquaterrários; além disso, há uma variedade de moldes, réplicas, esculturas e modelos didáticos. O MCN-SCB-UFPR possui também um terrário externo vegetado onde animais são mantidos para observação e desenvolvimento de atividades. A exposição permanente conta com material biológico exposto em balcões, vitrines além de painéis explicativos, com textos e imagens que auxiliam os visitantes a obterem as informações básicas sobre o material exposto. As visitações são monitoradas por bolsistas (do PCVAE) e voluntários treinados que abordam o tema principal da exposição, relacionando-o com questões envolvidas no cotidiano do público alvo. O tema estrutural da exposição aborda a divisão dos organismos em Cinco Reinos primeiramente proposto por WHITTAKER (1959), por ser a mais difundida no ensino básico. Para seguir a filosofia adotada, a visitação é iniciada com conhecimentos da área da Paleontologia com o propósito de oferecer aos alunos uma visão evolutiva da vida. Em seguida, são apresentados os organismos unicelulares e pluricelulares que compõem os grandes reinos de organismos (e.g monera, protistas, fungos, plantas, animais metazoários invertebrados e vertebrados). Esta monitoria corresponde a uma "aula" dinâmica, viva e interativa.

Além do monitoramento das visitações outras atividades também são desenvolvidas: produção de modelos, peças didáticas e organizações de coleções didáticas na área das Ciências Naturais para empréstimo a professores e instituições de ensino. O MCN-SCB-UFPR ainda tem um importante papel na formação acadêmica, possibilitando alunos de graduação e pós-graduação enriquecerem sua formação profissional e desenvolverem seus trabalhos de conclusão de curso, 
na orientação a professores do Programa de Desenvolvimento da Educação do Estado do Paraná (PDE-PR) e na disponibilização de cursos para a comunidade em geral.

Dentre as diferentes ações desenvolvidas pelo PCVAE estão incluídas palestras, cursos, mostras, exposições itinerantes, oficinas, programas de ensino e extensão em universidades, colégios praças e outros ambientes. Os cursos e oficinas oferecidas envolvem diferentes áreas do conhecimento: Parasitologia Humana, Biologia Celular, Genética, Zoologia, Botânica, Evolução, História da Ciência, Plantas tóxicas e medicinais, Fisiologia, Paleontologia, Riscos de automedicação, Farmacologia, Aspectos básicos sobre o Ensino de Ciências, Introdução ao Ofidismo, Aspectos gerais do ofidismo e das serpentes venenosas do Sul do Brasil; Reprodução, Princípios de Taxidermia, Proficiência básica em Ciências, Biologia experimental, Fotografia biológica, Física - Astronomia, Física - Eletromagnetismo, Física - Eletricidade Ótica, Matemática - Tangran, Matemática - Sistemas numéricos, Matemática - Teorema de Pitágoras, Matemática Esqueleto de Sólidos, Matemática - Geometria das dobraduras, Introdução a Informática, Aquarismo Didático, Biologia e Hereditariedade dos Insetos, Conhecendo as Aves, E os Peixes??? Nadam!!!, Biomas Paranaenses, O Homem do Avesso, Na Teia da Aranha, Herpetologia, Vermes Plathyhelmintes, Foraminíferos, entre outros. A duração destas oficinas pode variar de acordo com o propósito de cada uma delas, dependendo do tema e de sua profundidade.

Algumas das atividades do PCVAE não são realizadas dentro do $\mathrm{MCN}$ ou em espaços convencionais, mas em ambientes alternativos, por exemplo, utilizando-se do tempo despendido durante o deslocamento dos alunos para as atividades de extensão que pode ser utilizado para o desenvolvimento de atividades didáticas (e.g. a oficina "Ciência no Ônibus"), assim os discentes se familiarizam com os temas que serão desenvolvidos posteriormente.

O planejamento destas atividades envolve pesquisa e estudo de assuntos científicos e educacionais, preparação do material, execução e realização de avaliações pelo corpo de bolsistas e voluntários juntamente com a coordenação do Programa Ciência Vai à Escola. Essas avaliações são contínuas e periódicas contribuindo para verificar a efetividade das ações, acompanhando cada atividade ou ação executada.

Dentro do PCVAE, são realizadas dois tipos de avaliações: a avaliação direta, que é realizada pelo próprio programa; e a avaliação indireta que envolve os parceiros e outros participantes que não estão ligados diretamente. As avaliações diretas são realizadas após cada visitação ao MCN-SCB-UFPR, através de um questionário respondido pelo docente responsável pela turma e também são aplicados questionários sobre a aplicação de oficinas e cursos ministrados por integrantes do programa. Esses questionários são aplicados antes e depois das atividades propostas, a fim de verificar quantitativamente e qualitativamente se as informações transmitidas foram assimiladas pelo público alvo. As avaliações indiretas, como mencionado anteriormente são realizadas por parceiros participantes do programa, como, por exemplo, as 
avaliações realizadas pela Secretaria Estadual de Educação do Paraná durante os eventos "Educação Com Ciência" e "Fera Com Ciência" onde o PCVAE foi atuante.

\section{Resultados}

Ao longo dos 18 anos de atuação do MCN-SCB-UFPR e dos 15 anos do PCVAE, já foram atingidos mais de 120 mil participantes, 420 instituições de ensino da rede pública e privada em mais de 140 municípios. Os questionários aplicados periodicamente após as visitas guiadas mostram que menos de $10 \%$ dos participantes já haviam visitado um museu, de modo que os objetivos de popularizar e divulgar a Ciência estão sendo alcançados com sucesso. O público atingido por região no território brasileiro em visitações ao Museu de Ciências Naturais (MCNSBC-UFPR) e em cursos, palestras, oficinas, feiras e outros eventos aplicados pelo PCVAE encontra-se representado na Figura 1. Nesta, observa-se que a maioria do público atingido é de municípios do Sul do Brasil, sendo que o Estado do Paraná representa 66,7\% dos participantes da região, seguido de Santa Catarina e São Paulo, onde foram identificados visitantes de 23 e 20 municípios diferentes destes dois estados respectivamente. Esses resultados refletem a localização do MCN-SCB-UFPR, onde são desenvolvidas a maior parte das atividades, uma vez que este situa-se no estado do Paraná. Visitantes de países como EUA, Holanda, França, Costa Rica, Espanha e Chile também já foram recebidos pelo MCN-SBC-UFPR.

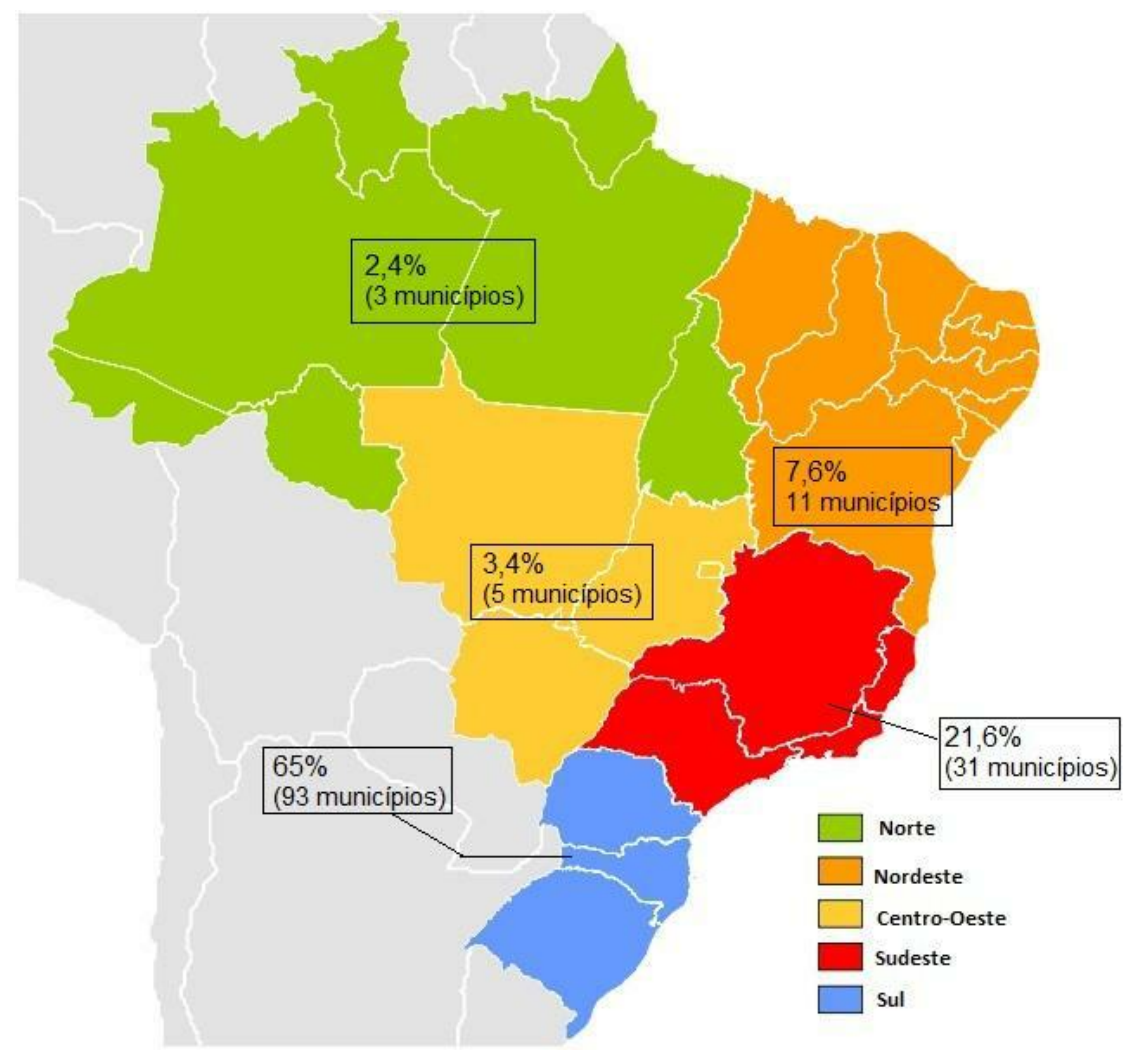

Figura 1 - Distribuição do público contemplado nas regiões brasileiras em visitação a exposição Os Seres Vivos no Ensino Fundamental e Médio" integrante do Museu de Ciências Naturais da UFPR (MCN-SBC-UFPR) e em aplicações de cursos, palestras, oficinas, feiras e outros eventos do PCVAE. 
Grande parte das atividades do PCVAE foram desenvolvidas no Município de Curitiba, entretanto, o programa já ministrou cursos, palestras e oficinas para municípios da região metropolitana e do interior do estado, como: Arapongas, Antonina, Campo Largo, Cerro Azul, Colombo, Dois Vizinhos, Guarapuava, Lapa, Londrina, Pinhais, Ponta Grossa, Tunas do Paraná e Umuarama.

A distribuição dos participantes do PCVAE ao longo dos anos é mostrada na Figura 2, onde é possível verificar que houve um crescimento de 2000 a 2006, atingindo o número máximo de participantes neste último ano, somando um total de 11.872 indivíduos. Entre 2007 e 2009, o programa teve uma média de 9.019 participantes por ano, com uma queda a partir de 2010.

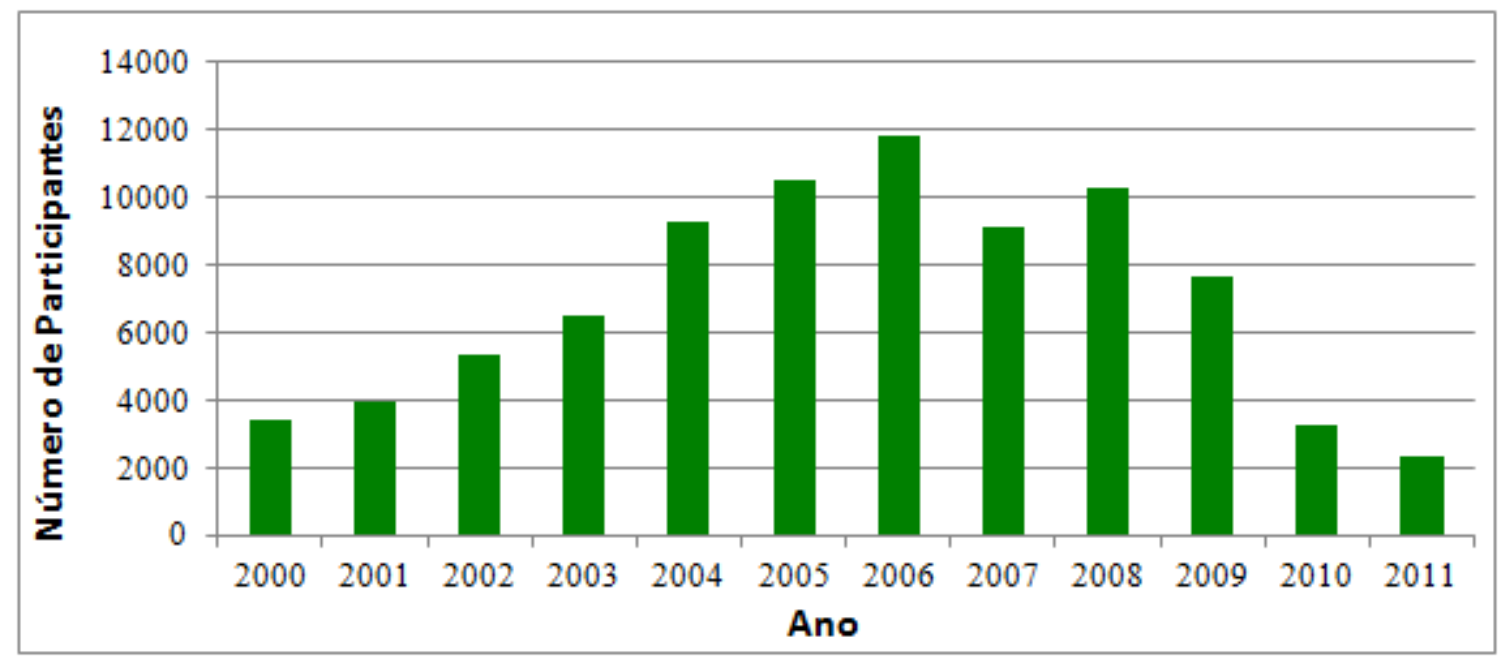

Figura 2 - Número anual de participantes do PCVAE entre os anos de 2000 a 2011.

O número anual de participantes ao longo dos anos de atividade tem oscilado em função de fatores internos da Universidade Federal do Paraná como greve nacional dos professores, servidores e alunos das universidades federais, mudanças curriculares em vários cursos de graduação, comprometendo a participação dos acadêmicos no PCVAE e também fatores internos ao MCN-SBC-UFPR; bem como, de fatores externos ao programa, como a reestruturação do currículo do Ensino Fundamental e Médio, da Rede Estadual de Educação do Paraná. Já o número de instituições de ensino, da rede pública e privada, contempladas no período de 2001 a 2011 ultrapassou 420 estabelecimentos, sendo a média anual de 65, entre escolas, colégios, projetos sociais, centros de educação infantil e pré-vestibulares, assim como universidades e faculdades (Figura 3). 


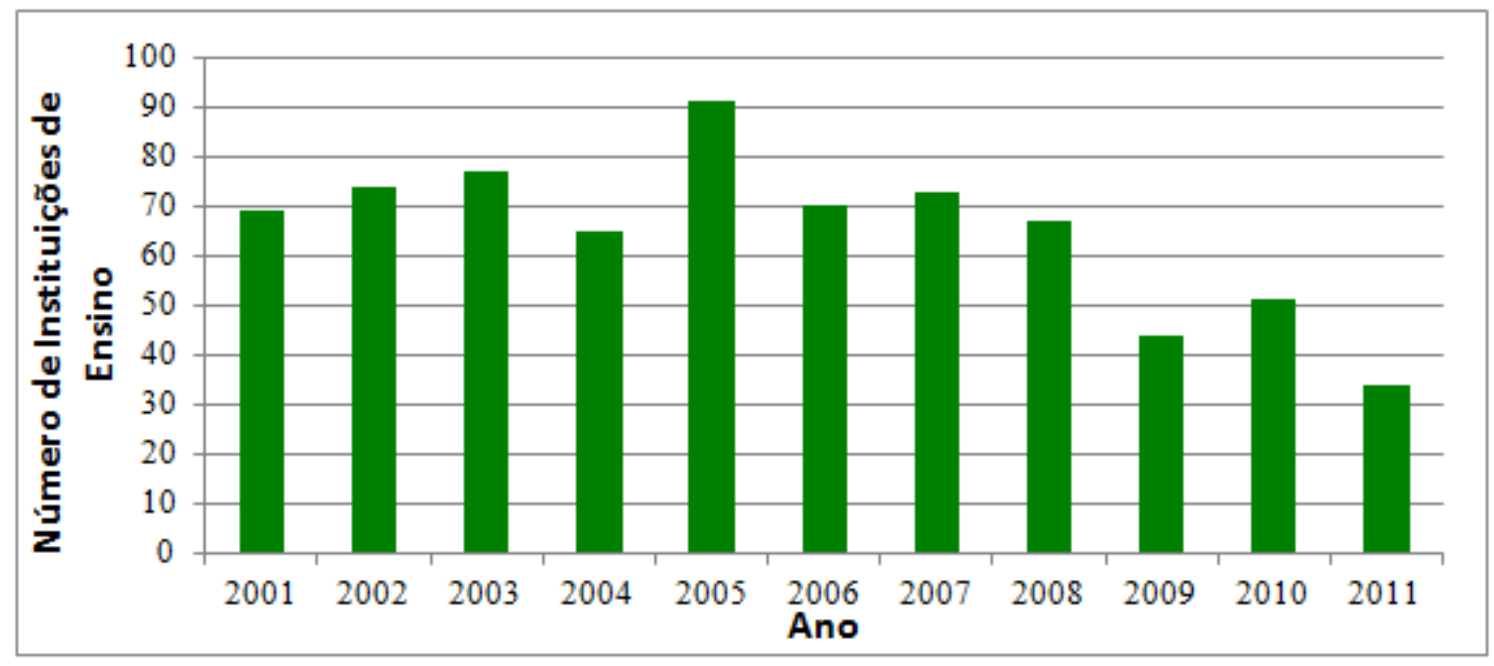

Figura 3 - Número de instituições de ensino contempladas anualmente pelo PCVAE entre os anos 2001 e 2011.

\section{Discussão \& Conclusão}

A divulgação científica no Brasil é uma atividade em permanente construção, principalmente quando se fala de ambientes de educação não formal como é o caso de museus e centros de Ciência e ainda mais quando o assunto é a educação básica. De acordo com BARROS (2002) a escola, que depende muitas vezes somente da presença do professor como mediador de informação, não tem as condições necessárias de atualizar-se na taxa que o mundo científico anuncia. Além disso, os professores usualmente utilizam-se como principal forma de ensino aulas expositivas, que além de privar a participação dos alunos, cansam os professores e os próprios discentes, o que muitas vezes não torna o processo de ensino/aprendizagem efetivo. Segundo KRASILCHIK (1987), uma das características do mau ensino das ciências é fazê-lo de forma expositiva, autoritária, livresca, mantendo os estudantes inativos, tanto intelectual como fisicamente.

De acordo com FREIRE-MAIA (2007), a Ciência pode ser visualizada sob dois aspectos fundamentais: a "Ciência-Disciplina" e a "Ciência-Processo". A primeira é a Ciência ensinada nas escolas, apresentada de uma forma pronta e acabada, que os alunos devem aprender para realizar exames e serem aprovados. É um conjunto de dados oferecidos muitas vezes de forma dogmática, sem demonstração de ideias opostas às dos livros didáticos, acarretando na aceitação de "verdades científicas", o oposto do que se espera na Ciência. Esta forma de ensino é chamada de tradicional por CANIATO (1997), e ele a caracteriza pela fala quase exclusiva do professor, alunos quietos, que ficam sentados praticamente imóveis anotando aquilo que ouvem. Além disso, o ensino formal não propicia a permanente atualização e formação que se fazem necessárias para que um profissional já formado e exercendo as suas funções na sociedade, 
principalmente na área de educação, possa manter-se inserido no processo de transformação social.

Já a "Ciência-Processo" segundo FREIRE-MAIA (2007) é a Ciência realizada pelo cientista, com uma metodologia adequada, que minimiza possíveis erros e permite que seja reproduzida por outro pesquisador igualmente qualificado, assim está constante reelaboração, ampliação e revisão. Esta pode ser dividida em duas fases: a própria pesquisa e a divulgação dos resultados. $O$ Programa "Ciência Vai à Escola" (PCVAE) visa conciliar a "Ciência-Disciplina" com a "CiênciaProcesso" acima mencionadas, aproximando o meio científico dos espaços escolares, articulando o ensino, a pesquisa e a extensão de forma expositiva, demonstrativa e interativa.

Por isso, consolidar, qualificar e ampliar a divulgação da Ciência é uma tarefa que deve ser transformada em um processo coletivo suficientemente amplo, que envolva instituições de pesquisa, universidades, comunicadores, cientistas, educadores, estudantes e o público em geral. Segundo CANDOTTI (2002) a maior responsabilidade que os cientistas e acadêmicos tem é a de educar para entender e transformar o mundo, tornando-o mais justo e igualitário.

Para que isso aconteça é necessário fugir do modelo de ensino-transmissão adotado pelas escolas e partir para uma educação que valorize a interação verbal, desencadeada por provocações, questões que estimulem os alunos a pensarem e manifestarem-se, de modo a aproximar o mediador (monitor) do estudante e este do conhecimento. Busca-se nesse método a reflexão do mundo, favorecendo ao aluno entrar em contato com as inúmeras leituras da natureza dentro de um compromisso com o cotidiano. Isto, sem esquecer das diversas "aventuras do saber" que o próprio aluno pode desbravar por si próprio e que não se limitam ao espaço de educação, mas que ele poderá experimentar e cada acontecimento do seu dia-a-dia e que jamais se esgotará.

Assim, para BARROS (2002) quando se fala em alfabetização científica, fica mais claro tratar da alfabetização de um indivíduo inserido socialmente, cumpridor do seu papel, da mesma forma que a não-discriminação de nenhum dos indivíduos socialmente ativos torna-se essencial para a proposta de uma sociedade democrática com a participação de todos os cidadãos no processo de construção social, em que o nível de informação deve satisfazer a todos. Desta forma, o professor possui como responsabilidade dentro de um processo cognitivo, criar situações que auxiliem na aprendizagem dos alunos, contanto que estas ocorram de forma autônoma e condizente com o estilo e característica individual de cada um (KRASILCHIK, 2004). Estas são as propostas do PCVAE, que visa divulgar a Ciência abordando temáticas mais práticas do que expositivas, atraindo a atenção dos estudantes de forma interativa para cada um dos temas abordados.

A interatividade permite encurtar a distância existente entre o docente e os discentes, facilitando o contato entre ambas as partes, levando à reflexão que possibilita empreender um 
método de indagação orientada atingindo uma maior metacognição, compreensão e realização pessoal. Essa prática se torna um desafio tanto aos estudantes quanto aos professores. Assim o desenvolvimento da docência não ocorre de forma isolada, mas se torna um processo compartilhado por toda comunidade educacional (alunos e professores), considerando todos como aprendizes (BAIRD et al., 1991).

Por ser um programa de longa duração, no PCVAE as ações não são aplicadas como uma forma pontual de atender a necessidades de conhecimentos específicos em uma determinada área, mas como uma postura científica frente ao ensino de Ciências. Isso aliado ao Método Científico utilizado de forma interativa tem mostrado através das avaliações uma aquisição considerável de conhecimento. Os resultados obtidos pelo Programa Ciência vai à Escola demonstram que a filosofia e a metodologia empregadas vem cumprindo satisfatoriamente os objetivos de educar, popularizar, alfabetizar e divulgar a Ciência.

\section{Agradecimentos}

Os autores agradecem ao Professor Euclides Fontoura da Silva Junior (in memorian) que foi o principal idealizador e coordenador do PCVAE (1997-2010), além de ter dirigido o MCN-SCBUFPR durante dezesseis anos (até 2010), cuja iniciativa e satisfação pela popularização, alfabetização e divulgação científica foi levada até os últimos dias de sua vida. Agradecem também aos colaboradores, estagiários e voluntários e a todas as pessoas que tem participado do Programa Ciência Vai à Escola tornando este sonho possível; a Pró-Reitoria de Extensão e Cultura da UFPR (PROEC-UFPR), Pró-Reitoria para Assuntos Estudantis da UFPR (PRAE-UFPR), Fundação Araucária e a Sociedade Brasileira para o Progresso da Ciência (SBPC) pelo apoio prestado durante estes anos. Agradecem ainda a acadêmica Flávia Schlichta na elaboração das figuras aqui utilizadas. 


\section{Referências}

BAIRD J.R.; FENSHAM, P.J.; GUNSTONE, R.F. \& WHITE, R.T. The importance of reflection in improving science teaching and learning, Journal of Research in Science Teaching, v.28, n.2, p.163182, 1991.

BARROS, H. L. A Cidade e a Ciência. In: MASSARANI, L.; MOREIRA, I. C.; BRITO, F. orgs.). Ciência e público: caminhos da divulgação científica no Brasil. Rio de Janeiro: Casa da Ciência - Centro Cultural de Ciência e Tecnologia da Universidade Federal do Rio de Janeiro: Forum de Ciência e Cultura, 2002. 302 p.

CANDOTTI, E. Ciência na Educação Popular. In: MASSARANI, L.; MOREIRA, I. C.; BRITO, F. Ciência e público: caminhos da divulgação científica no Brasil. Rio de Janeiro: Casa da Ciência - Centro Cultural de Ciência e Tecnologia da Universidade Federal do Rio de Janeiro. Forum de Ciência e Cultura, 2002. $302 \mathrm{p}$.

CANIATO, R. Com ciência na educação: Ideário e prática de uma alternativa brasileira para o ensino da Ciência. Campinas: Papirus, 1997. 127 p.

CORRÊA, D. M. V. B.; \& SILVA-JUNIOR, E. F. 2010. Ciência Vai à Escola: o lúdico na educação em Ciências. Disponível em: http://www.diaadiaeducacao.pr.gov.br/portals/pde/arquivos/13698.pdf. Acesso em: 06/05/2012.

FREIRE-MAIA, N. A Ciência por dentro. 7a Ed: Petrópolis: Vozes, 2007. 213 p.

KRASILCHIK, M. O professor e o currículo das ciências. São Paulo: EDUSP, 1987. 80 p. KRASILCHIK, M. Prática de Ensino de Biologia. 4ạ ed: São Paulo: EDUSP, 2004. 197 p.

SILVA-JUNIOR, E.F. Museu de Ciências Naturais da UFPR - o "Nascer" de um Museu. In: Crestana, S.; Hamburger, E.W.; Silva, D.M. \& Mascarenhas, S. (orgs.) Educação para a Ciência: Curso para Treinamento em Centros e Museus de Ciência. São Paulo: Editora Livraria da Física, 2001. p. 549554.

SILVA-JUNIOR, E.F. Programa Ciência Vai à Escola: Relatório Anual de Programa de Extensão Universitária. Curitiba: UFPR, 2007. 353 p.

WHITTAKER, R.H. On the broad classification of organisms. The Quartely Review of Biology, Chicago, v.34, p. 210-226. 1959.

Victor E. Pauliv1,2 -; biomuseu@ufpr.br 1Programa de Pós Graduação em Geociências Universidade Federal do Rio Grande do Sul Porto Alegre - Rio Grande do Sul 2Museu de Ciências Naturais - Setor de Ciências Biológicas - Universidade Federal do Paraná Curitiba - Paraná victorpauliv@hotmail.com

Luciana C. Carvalho2 - carvalho.c.lu@gmail.com

Camilla Felippe2 - camilla.felippe@yahoo.com.br

Rafaela Bobato2 - rafaelabobato@gmail.com

Fernando A. Sedor2 - sedor@ufpr.br 